\title{
La pandémie de COVID-19 et le débat sur l'étalement urbain : tournant majeur ou accident de parcours?
}

\author{
Martin Simard ${ }^{\mathrm{a}}$
}

RÉSUMÉ. Le 4 mars 2020, le magazine L'Actualité publiait un article soulignant la tendance à la densification et à la diversification des espaces urbains au Québec. Moins de deux semaines plus tard, la population québécoise était appelée au confinement à la maison à cause de la pandémie de COVID-19. Cette crise majeure présente la maison individuelle comme l'ultime refuge pour se protéger du monde extérieur, remettant potentiellement en cause 30 ans de lutte contre l'étalement urbain et ses conséquences environnementales. Ce texte résume le débat entre deux écoles de pensée qui s’opposent sur les conséquences à long terme de la pandémie sur l'aménagement et l'urbanisme au Québec.

\begin{abstract}
On March 4, 2020, L'Actualité magazine published an article bighlighting the trend towards densification and diversification of urban spaces in the province of Québec. Less than two weeks later, the Quebec population was called to lock up at home because of the COVID-19 pandemic. This major crisis presents the detached house as the ultimate refuge to protect oneself from the outside world, potentially calling into question 30 years of the fight against urban sprawl and its emvironmental consequences. This text summarizes the debate between two schools of thought that clash over the long-term consequences of the pandemic on urban planning in Quebec.
\end{abstract}

\section{Introduction}

Le 4 mars 2020, le magazine québécois L'Actualité publiait un article s'intitulant « Vivre à la verticale ». Ce texte bien ficelé souligne la tendance à la densification et à la diversification des nouveaux espaces résidentiels, notamment dans les régions métropolitaines de recensement (RMR) de Québec et de Montréal :

Afin de contrer l'étalement urbain, de favoriser le transport collectif et de plaire à la portion non négligeable de consommateurs chez qui l'appel de la maison individuelle ne résonne plus, des zones urbaines sous-utilisées comme les parcs industriels en perte de popularité, les centres commerciaux désuets et les stationnements de surface - sont transformées en ce que les urbanistes appellent des quartiers intégrés mixtes. (Diotte, 2020, s. p.)
Cet article témoignait (il semble pertinent de parler au passé) d'une certaine revalorisation de l'urbanité au Québec après des décennies de triomphe de la banlieue pavillonnaire ${ }^{1}$ comme milieu de vie, tel que l'a mis en exergue le film canadien C.R.A.Z.Y. ou la série américaine Beautés désespérées. Moins de deux semaines plus tard, les écoles, les commerces et les industries étaient progressivement fermés, et la population québécoise était appelée au confinement à la maison par les plus hautes autorités, comme dans le reste du pays et ailleurs dans le monde. Le responsable : le virus COVID-19, dont la pandémie paralyse toujours la société au moment d'écrire ces lignes. Tout comme les grandes épidémies ayant frappé le monde, de la peste bubonique dans l'Europe du XIVe siècle jusqu'au typhus (1832) ou la variole (1885) dans le Québec du XIXe siècle, sans compter la fameuse grippe espagnole de 1919, la pandémie se répand plus rapidement et plus fortement dans les milieux urbains (Goulet, 2020). En conséquence, la grande ville et ses quartiers anciens de moyenne et forte densités sont depuis longtemps

${ }^{\text {a }}$ Professeur et membre du Centre de recherche sur le développement territorial (CRDT), Université du Québec à Chicoutimi 
synonymes de saleté, de promiscuité et de maladie dans l'imaginaire collectif.

Bien que la COVID-19 soit un virus certes très contagieux, mais beaucoup moins dangereux que ceux cités plus avant, et que nous soyons mieux pourvus en matière d'hygiène et de services de santé, il convient de se questionner sur ses effets à moyen et long termes sur le plan de l'occupation du territoire. En effet, plusieurs interrogations s'imposent dans le champ de l'aménagement et de l'urbanisme : Est-ce que l'animation des zones centrales et les événements de foule reprendront leur vigueur habituelle? Le télétravail affectera-t-il le marché des immeubles de bureaux et, par ricochet, la vitalité des centres-villes, voire des pôles d'emplois situés en banlieue? Finalement, est-ce que le relativement timide retour des dernières années vers les moyenne et haute densités résidentielles se maintiendra ou assisterons-nous à une nouvelle vague d'étalement urbain?

Nous nous intéresserons particulièrement à la dernière question, ce qui exige d'abord un rappel des grands éléments du débat sur l'étalement urbain, lequel débat est en arrière-plan des théories et pratiques urbanistiques depuis une quarantaine d'années.

\section{L'étalement urbain : un débat récurrent sur notre mode de vie}

L'étalement urbain est un terme péjoratif qui désigne l'urbanisation diffuse des villes nordaméricaines actuelles, forme d'aménagement qui tranche avec les villes traditionnelles ou les quartiers construits avant la Seconde Guerre mondiale. L'expression peut être définie comme étant l'expansion démesurée du tissu urbain reliée principalement aux zones d'habitation de basse densité (Simard, 2014). L'idée de démesure est centrale lorsque l'on parle d'étalement urbain. C'est-à-dire que la croissance de l'espace urbain est largement supérieure à la croissance démographique. Donc, chaque personne occupe en moyenne plus d'espace que par le passé pour se loger, mais aussi pour se déplacer, faire des achats ou se divertir.

Associée à la montée de la classe moyenne, à l'amélioration de la qualité de vie et à la sécurité des enfants, l'urbanisation diffuse a des conséquences certaines, en particulier sur les plans environnemental et économique (voir tableau 1). Quoi qu'il en soit, la maison individuelle et son voisinage verdoyant sont puissamment ancrés dans le mythe de la réussite, et ils constituent une part importante du rêve américain :

C'est une sorte de marque de fabrique nordaméricaine : lorsque l'on survole en avion une ville du Canada ou des États-Unis, juste avant l'atterrissage, on voit souvent [les maisons individuelles] s'aligner, presque à perte de vue. Machinalement posées, les unes à côté des autres, séparées au centimètre près, comme un motif géométrique dupliqué à l'infini ». (Desgré, 2012, s. p.) ${ }^{2}$

Ce rêve américain est aussi un rêve canadien et québécois. L'engouement des Québécois pour la maison individuelle peut être considéré comme une forme banale d'américanisation du mode de vie, alors que certains y voient plutôt un lien avec nos racines rurales (Fortin, Després et Vachon, 2002). D'autres perçoivent des traits originaux à la maison unifamiliale québécoise, traits qui en feraient même une sorte de patrimoine, du moins les bungalows des années 1960 et 1970 (Morriset et Noppen, 2004). Ainsi, les points de vue sont partagés au sujet de l'aménagement type des espaces résidentiels de la Belle Province (voir figure 1).

\begin{tabular}{|l|l|}
\hline Environnement & Perte de terres agricoles ou boisées et GES liés aux transports \\
\hline Économie & Coûts d'infrastructures de l'État et coûts de transport des ménages \\
\hline Société & Individualisme, surconsommation et séparation des groupes sociaux \\
\hline Urbanité & Séparation des activités urbaines et création de no man's lands dans la ville \\
\hline Santé & Décourage la marche, favorise la sédentarité et l'obésité \\
\hline
\end{tabular}

Tableau 1 - Conséquences néfastes de l'étalement urbain 


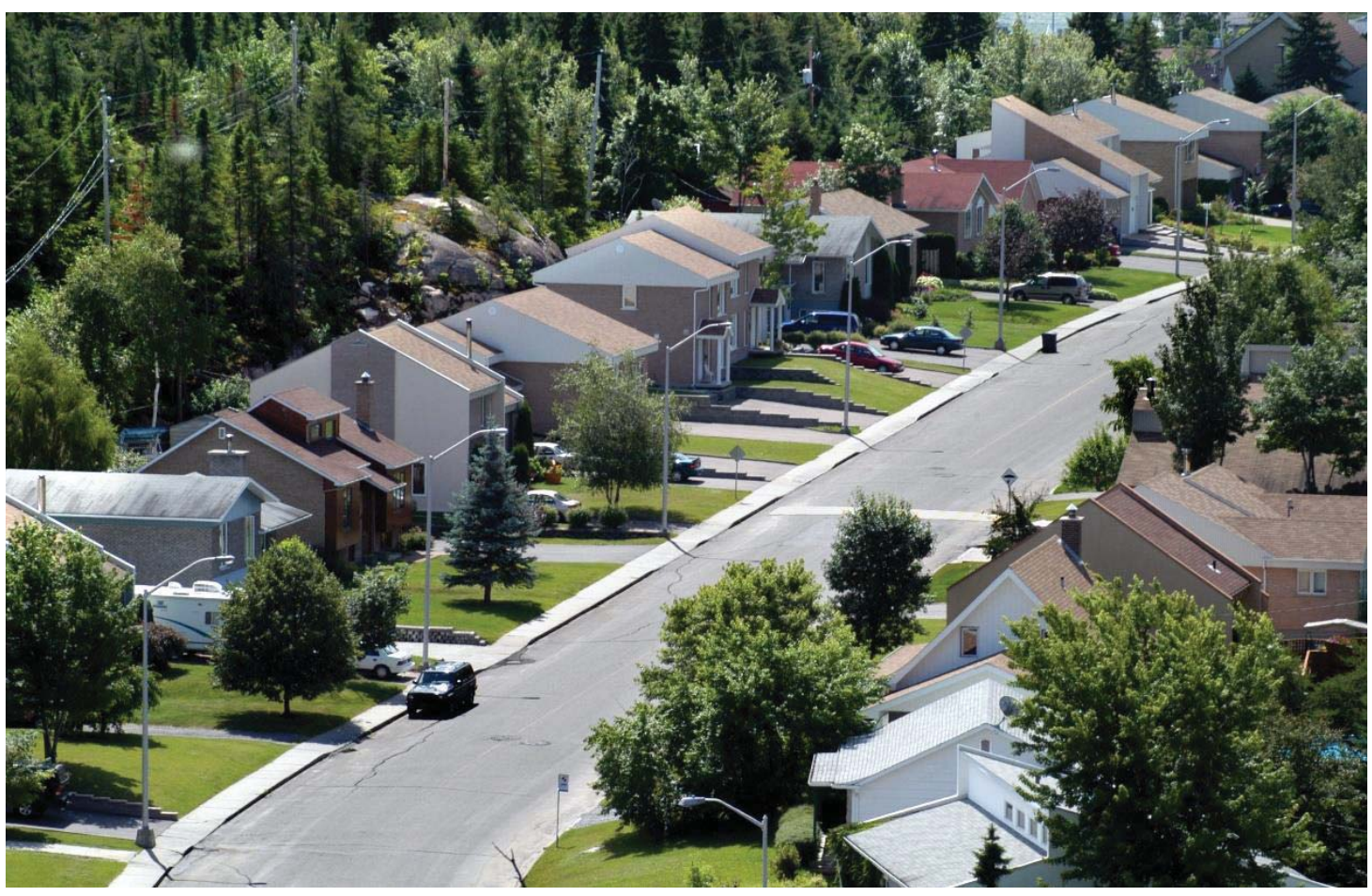

Figure 1 - Quartier pavillonnaire d'un arrondissement de Saguenay Source : Ville de Saguenay, https://batissons.saguenay.ca)

Au-delà des discussions sur l'origine de l'intérêt des Québécois pour la maison unifamiliale ou sur son caractère original ou non, l'étalement urbain donne lieu à un débat idéologique important. Pour les tenants de la libre entreprise et des droits individuels à tout prix, l'étalement urbain, voire l'établissement des urbains en campagne est le simple résultat de la conjonction des valeurs culturelles, des possibilités de mobilité, de l'offre du marché, en plus de constituer une occasion de développement économique axée sur la marchandisation du sol et du milieu bâti (Cox, 2006). En réalité, on ne vend pas que de la brique et du béton; on vend aussi du rêve, comme l'indiquent fréquemment les noms des développements domiciliaires: Jardins du bonheur, Faubourg du boisé, Domaine des oiseaux bleus, etc.

Le message subliminal du marché est le suivant: chacun mérite ce qu'il y a de mieux en ce qui a trait à l'habitation ${ }^{3}$, selon ses goûts et sa capacité de payer, peu importent les conséquences collectives, qu'on rejette du revers de la main. Dans la psyché nord-américaine, tout individu ou ménage aspire à bâtir maison et qui pourrait l'empêcher sur un territoire vaste et relativement peu peuplé, sinon un État malveillant ou totalitaire? D'ailleurs, pour faire un peu d'ironie, il est de notoriété publique qu'on ne peut élever d'enfants décemment en dehors de ces quartiers peuplés de bonnes familles, en retrait du tourbillon de la ville.

À contrario, pour la plupart des spécialistes du territoire (géographes, urbanistes, etc.) et les militants environnementalistes, les impacts de l'urbanisation diffuse doivent conduire le gouvernement provincial et les administrations locales à limiter l'étalement urbain. Sous ce regard critique, ce fléau des temps modernes crée notamment « une distanciation sociale » entre les habitants moins fortunés des quartiers anciens et ceux plus aisés de la banlieue pavillonnaire (Jargowsky, 2002). Les conséquences environnementales sont également importantes, à tel point que les ressources de la planète ne seraient pas suffisantes pour supporter l'empreinte écologique de ce mode de vie, si les populations de tous les pays étaient en mesure de se le permettre financièrement (WWF, 2014).

En outre, des observateurs plus radicaux voient même dans l'étalement urbain un complot d'envergure mondiale orchestré par les grandes entreprises immobilières, pétrolières et automobiles, lesquelles y trouvent leurs profits. Qu'il suffise de mentionner que la demande en pétrole est bien assurée dans la 
durée lorsque des millions de Nord-Américains doivent parcourir quotidiennement des dizaines de kilomètres pour se rendre au travail, soit un navettage d'une durée moyenne de 26,9 minutes (Saldivia, 2018) ${ }^{4}$. Ce complot des grands acteurs économiques soutenus par les États, lesquels construisent des autoroutes et autres aménités, aurait des répercussions géopolitiques et militaires considérables, par exemple au Moyen-Orient (Gonzalez, 2010).

Ce débat presque sans issue montre le paradoxe de la coexistence des désirs légitimes des ménages et des effets collectifs néfastes dans les sociétés de grands nombres. Dans ce contexte, les gouvernements n'ont jamais vraiment voulu intervenir trop directement, sauf dans le cas de la Loi sur la protection du territoire et des activités agricoles du Québec. Malgré ses atouts, cette loi affiche de nombreuses limites pour véritablement freiner l'étalement urbain (Côté, Mercier et Roy, 2014). Reste alors la solution de la sensibilisation aux effets de l'urbanisation diffuse et des campagnes de valorisation de la vie urbaine, notamment sous l'angle du développement durable et des changements climatiques.

C'est cette stratégie à long terme, brillamment mise en œuvre au Québec par l'organisme Vivre en ville, qui est remise en question par l'émergence de la COVID-19, par l'imposition du confinement, par la promotion de la distance physique entre les individus et par le port du masque.

\section{Le virus COVID-19 : quels effets sur l'urbanité?}

Après cette mise en contexte du débat sur l'étalement urbain, il est maintenant temps de revenir à notre question principale: Est-ce que la tendance des dernières années vers une augmentation des densités résidentielles se maintiendra ou assisterons-nous à une nouvelle vague d'étalement urbain au Québec? Nous tenterons d'y répondre à l'aide d'une revue de presse $^{5}$.

Il convient d'abord de dire que la situation québécoise ne parait pas différente de celle du reste de
l'Amérique à cet égard, si ce n'est que le virus a frappé plus fortement ici que dans les autres provinces et territoires canadiens ou dans la majorité des États américains (Deglise, 2020). Montréal a été particulièrement affectée par le virus et la région métropolitaine de recensement du même nom fait l'objet de mesures spécifiques de la part du gouvernement du Québec (Bruemmer, 2020).

De manière plus générale, le choc est important aux échelles continentale et mondiale. Au même titre que lors des attentats du 11 septembre 2001 à New York ou de la crise économique mondiale de 2008, plusieurs observateurs et analystes sont prompts à dire que les choses ne seront plus jamais pareilles après la pandémie de COVID-19. Dans quelle mesure cette affirmation est-elle juste? Personne ne le sait vraiment à l'heure actuelle.

Il est vrai que le confinement obligatoire à la maison, l'arrêt d'une grande partie des activités industrielles et commerciales ainsi que la fermeture des frontières pour une période de plusieurs mois, avec de possibles récidives, sont de nature à bouleverser un très fort nombre de gens. Ces changements drastiques du quotidien et l'effondrement de l'économie s'accompagnent de la peur de l'autre, devenu soudainement un potentiel porteur de virus, soit un danger à éviter plutôt qu'une personne qu'on gagnerait à connaître ou qu'il ferait bon de côtoyer (Guillemette, 2020).

Une conséquence majeure de tout ceci est sans doute la signification nouvelle, ou renforcée, de la maison perçue comme l'ultime refuge pour se protéger du monde extérieur (voir figure 2). De manière plus pratique, l'habitation devient aussi un lieu de travail sécuritaire auquel on accède sans déplacement, ce qui au demeurant permet de prendre des pauses pour manger, se reposer ou effectuer des tâches ménagères. La maison de banlieue est aussi proche des grandes chaînes commerciales bien approvisionnées qui ont résisté à la fermeture (Costco, Walmart, etc.). Est-ce que ces changements « habitationnels » perdureront? 


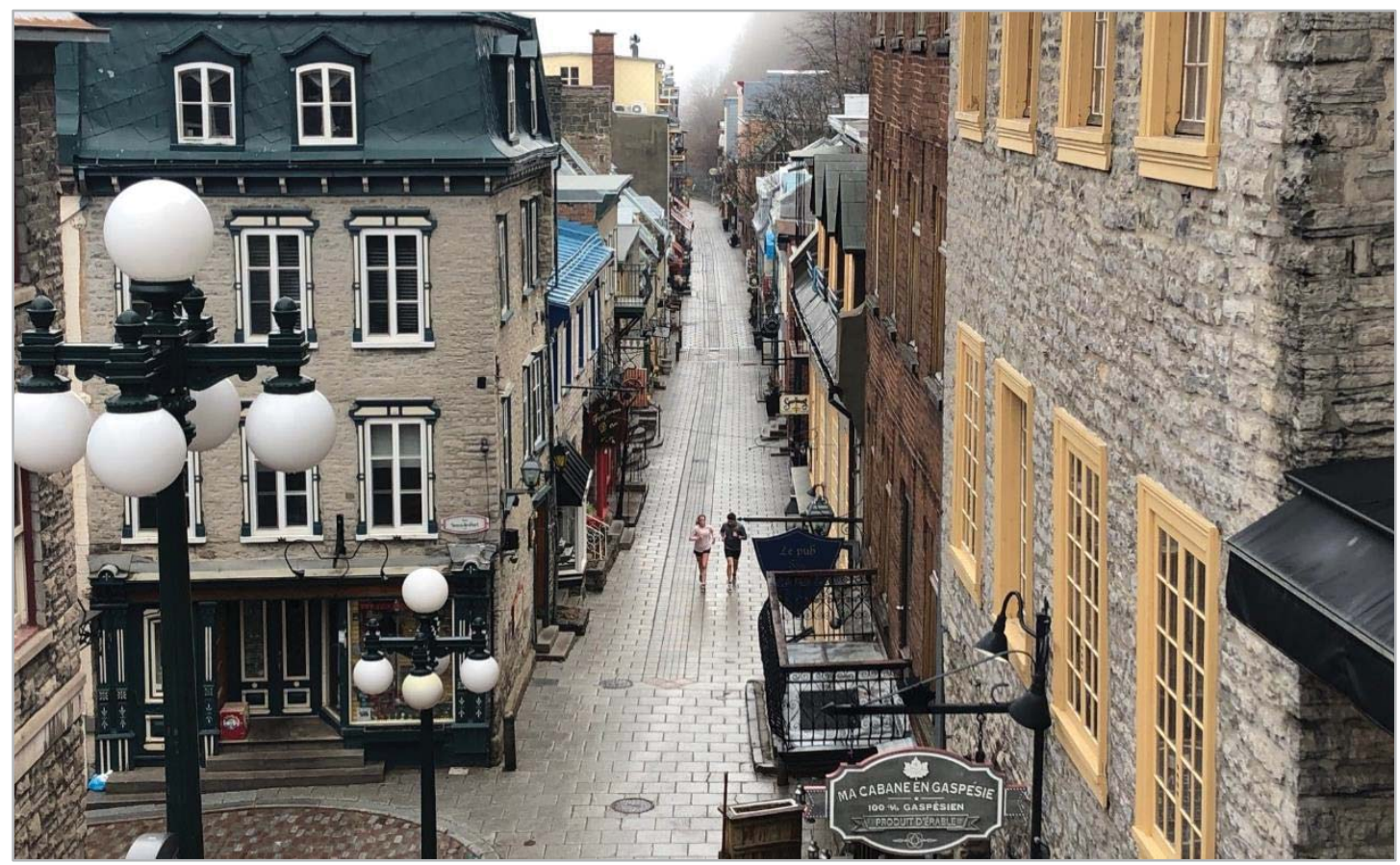

Figure 2 - La rue du Petit Champlain à Québec au moment du confinement Source : Martin Simard, 2020

Le bilan que nous pourrons faire des effets sociopsychologiques et sanitaires de la pandémie de COVID-19 dans deux, trois ou cinq ans déterminera l'ampleur des conséquences de celle-ci sur l'aménagement et sur la vitalité des villes de taille moyenne ou supérieure. Selon notre interprétation, deux écoles de pensée s'affrontent sur le sujet: ceux et celles qui croient que les multiples intérêts de la socialisation triompheront de la peur, groupe à distinguer de ceux et celles qui entrevoient l'avènement durable de comportements plus ou moins agoraphobiques. Appelons respectivement celles-ci l'école de la proximité et l'école de la distance.

Pour les tenants de la première école de pensée, le caractère grégaire de l'humain reviendra au galop :

L'histoire démontre en fait que les villes ont la couenne dure. Ni les épidémies, ni les bombardements n'ont jusqu'ici pu convaincre les humains de cesser de se rassembler. Les forces de la densité - économies d'échelle, synergies de proximité, déplacements raccourcis, accès aux services, etc. - vont demeurer des atouts dans le monde de demain. (Savard, 2020, s. p.)
Les habitudes auraient, semble-t-il, la vie dure :

Après une crise importante, au lieu de repenser complètement l'aménagement, on a tendance à refaire comme avant, mais plus solide, pour montrer qu'on est résilient. (C. Poitras, citée par Cambron-Goulet, 2020, s. p.)

Au-delà de la volonté de migrer vers le péri-urbain, il faut également considérer la capacité financière des ménages :

En fait, très peu de gens sont à même de déménager du jour au lendemain. Peut-être que quelques personnes qui ont les moyens de déménager le feront, mais ce n'est pas facile. (F. Paulhiac, citée par CambronGoulet, 2020, s. p.)

À l'opposé de cette lecture plutôt optimiste du retour à la normale, l'école de la distance propose un autre discours, tant pour vanter la maison individuelle, voire la campagne, que pour dénoncer le non-accès aux logements de qualité pour une partie de la population. De la sorte, selon les témoignages d'agents immobiliers américains rapportés par le USA Today, on peut déjà percevoir une volonté de quitter la grande ville chez une partie de la clientèle (Menton, 2020). 
Un journaliste présente simplement ce que semble ressentir de nombreuses personnes :

La crise sanitaire étant vécue plus confortablement dans les maisons que dans les appartements, certains estiment que ce marché verra affluer une nouvelle demande. (Litzier, 2020, s. p.)

En France, où la densité résidentielle est généralement plus forte de même que les clivages sociaux, l'assignation à résidence est vécue différemment selon la classe sociale :

Le confinement est un concept de bourgeois. Cela implique de posséder une maison bourgeoise dans laquelle se retirer. Ça ne correspond pas du tout à la réalité [des grands ensembles français]. (H. Esmili, cité par Hummel, 2020, s. p.)

En arrière-plan de ces prédictions, la question du rôle de la densité résidentielle dans la transmission du virus se révèle centrale. Comme le prétend un analyste :

Clearly densification is and has been the problem with some of this. COVID-19 puts a fundamental challenge to how we manage urbanization. (Klaus, 2020, s. p.)

Les comparaisons du nombre de cas de COVID-19 entre Québec et Montréal ou la mise en relation des situations de Los Angeles et New York donnent ainsi des munitions aux « distancialistes » :

Alors que la pandémie a explosé à New York, avec plus de 10000 morts et des services d'urgence et des morgues débordés, la situation est restée contenue en Californie. [...] La densité de population et l'urbanisation extrême de New York peuvent en partie expliquer ce lourd bilan. (Banguet, 2020, s. p.)

Dans la même veine, Dre Mylène Drouin, directrice régionale de la santé publique de Montréal, affirme ceci à propos du secteur de Montréal-nord :

Il existe un enjeu de capacité de maintenir la distanciation sociale. On le sait, à cause de la densité urbaine, beaucoup de gens ont de la difficulté à la respecter - il y a peu de parcs, peu d'espaces dans les rues. C'est la même chose dans les commerces. (La Presse canadienne, 2020, s. p.)

Néanmoins, d'autres cas de figure, comme Hong Kong ou Singapour, vont dans le sens contraire du lien directement proportionnel entre la densité de population et le nombre de personnes atteintes de la COVID-19. (Alter, 2020)

Ainsi, un observateur plus nuancé sur la nature virale de la densité urbaine laisse toutefois présager une popularité renouvelée de la maison individuelle, donc la relance possible de l'étalement urbain :

Les villes ont été largement montrées du doigt dans cette crise en pointant le fait qu'elles étaient des territoires plus denses que les autres. En réalité, la densité, ce n'est pas un facteur réel d'explication du virus. C'est évidemment des lieux où il y a plus d'interaction ou de connexions, donc ça a favorisé la dispersion du virus. Le véritable allié du virus aujourd'hui, c'est la suroccupation, la promiscuité, notamment à l'échelle du logement. (R. Rivaton, cité par Leymarie, 2020, s. p.)

Au final, quelle que soit l'ampleur des impacts sur la forme urbaine, bon nombre de petites habitudes pourraient être transformées dans l'ère de l'aprèspandémie, tant au centre-ville qu'en banlieue :

Le jour approche peut-être où on voudra prendre notre température ou nos signes vitaux à l'entrée des salles de spectacles, dans les transports collectifs ou les lieux publics. Cela risque de changer le rythme des villes. Les mouvements seront plus lourds, plus compliqués. On verra peut-être des files d'attente là où il n'y en avait jamais eu. Comme depuis quelques jours aux portes des épiceries et des pharmacies. Vivra-t-on dans l'insécurité chaque fois que quelqu'un toussera au théâtre ou dans la rue? Va-t-on commencer à se méfier en permanence les uns des autres, à se surveiller, se dénoncer? (Bourque, 2020, s. p.)

\section{Conclusion}

Au-delà des écoles de pensée, si la peur de l'autre et l'appel du foyer pour le télétravail et les loisirs persistent dans de fortes proportions, les retombées urbanistiques seront nombreuses. En discutant des 
travaux publics du passé sur les aqueducs et les égouts, lesquels ont suivi différentes épidémies, Ian Klaus (2020) utilise l'expression «assainissement à l'aide d'infrastructures numériques $»^{6}$ pour exprimer le rôle que sont appelés à jouer les réseaux sociaux, l'Internet et le télétravail dans les années à venir.

Ces retombées pourraient constituer un recul de plus de 30 ans, en particulier en matière de dynamisme des centres-villes et de lutte à l'étalement urbain. Pourquoi? Parce qu'il est évident que le confinement ou le réflexe de passer plus de temps au foyer favorise l'acquisition de maisons individuelles, idéalement de grand gabarit. En effet, celles-ci offrent plus d'espace intérieur pour se replier et plus d'espace extérieur pour " prendre l'air » à l'abri du danger, pour jardiner, pour bricoler ou pour rencontrer quelques amis triés sur le volet.

En parallèle au possible mouvement en faveur de l'urbanisation diffuse, les espaces centraux des agglomérations pourraient subir une baisse d'attrait et d'achalandage parce qu'ils concentrent les espaces ludiques et les lieux de travail marqués par le rassemblement et la proximité d'une foule d'individus de tous horizons. Si ce n'est pas une vague de fond, il y aura certainement des modifications sensibles : trottoirs plus vastes, rues réservées aux piétons, restaurants à faible capacité axés sur les commandes à livrer, festivals et événements sportifs moins nombreux et plus modestes, transports en commun sous-utilisés, etc.

Néanmoins, parler de désurbanisation et de mouvements de populations vers les régions périphériques paraît excessif (Saint-Hilaire, 2020). Les changements se feront aux échelles métropolitaine ou infrarégionale et non entre les régions, à notre avis. Il y a donc peu de chance que les travailleurs montréalais déménagent à Saguenay, par exemple. C'est plutôt les villages voisins de Saguenay qui risquent d'attirer de nouveaux ménages provenant de cette ville moyenne.

Tout ne sera pas négatif dans la vie urbaine de l'après-pandémie. Le télétravail devrait limiter les embouteillages, plus de gens devraient aménager un potager ou s'alimenter auprès de producteurs locaux ou régionaux, alors que le tourisme de proximité est appelé à prendre de la vigueur par rapport aux destinations exotiques. Selon les urbanistes montréalais Raphaël Fischler et Sylvain Gariépy, interviewés récemment :

La pandémie doit surtout pousser à repenser ses habitudes de vie en milieu urbain ainsi que l'aménagement des espaces publics pour les rendre plus attrayants et cohérents avec les mesures de santé publique en temps de crise sanitaire. (Caillou, 2020, s. p.)

Espérons qu'il ne s'agisse pas là de vœux pieux et que les innovations urbanistiques à venir soutiendront l'attrait de l'urbanité et un certain contrôle sur l'étalement urbain. La bonne qualité et une plus grande superficie des logements dans les zones urbaines centrales seront aussi à promouvoir pour maintenir compétitif ce secteur du marché immobilier et permettre des centres-villes habités et dynamiques.

\section{NOTES}

1 L'expression « banlieue pavillonnaire » vise à distinguer les banlieues nord-américaines aisées et de basse densité résidentielle par rapport aux banlieues françaises, caractérisées par les grands immeubles et certains problèmes sociaux.

2 Cette auteure défend plus tard dans ce texte l'idée qu’il existe une certaine diversité architecturale dans les banlieues pavillonnaires.

3 Ce « mieux » est évidemment un construit social, et non un fait objectif.

4 Cette dernière donnée s'applique pour les États-Unis uniquement, mais on peut penser que la situation est semblable au Canada.

5 Lorsque cet article a été écrit, il n'y avait pas encore d'articles scientifiques ou de livres disponibles traitant précisément de la pandémie de COVID-19 et de ses impacts sur les villes et l'urbanisme.

6 L'expression anglaise employée est la suivante : Digital infrastructure might be the sanitation of our time.

\section{RÉFÉRENCES}

Alter, L. (2020, 25 mars). Urban density is not the enemy, it is your friend. Blogue Tree Hugger. Repéré à www.treehugger.com/ urban-design/urban-density-not-enemy-it-your-friend.html 
Banguet, L. (2020, 15 avril). En Californie, la distanciation sociale a fait la différence face au coronavirus. La Presse. Repéré à www.lapresse.ca/international/etats-unis/202004/15/01-5269395-en-californie-la-distanciation-sociale-a-fait-ladifference-face-au-coronavirus.php

Bourque, F. (2020, 4 avril). Comment la pandémie va changer les villes. Le Soleil. Repéré à www.lesoleil.com/chroniques/francoisbourque/comment-la-pandemie-va-changer-les-villes-0e55d77e635a6ddd497c1eecead14976

Bruemmer, R. (2020, 3 avril). Montreal is the epicentre of the pandemic, public health director says. Montreal Garette. Repéré à https:// montrealgazette.com/news/local-news/montreal-hit-by-rapid-rise-in-number-of-covid-19-cases/

Caillou, A. (2020, 14 avril). Les atouts de la ville mis à mal par la pandémie. Le Devoir. Repéré à www.ledevoir.com/societe/transportsurbanisme/576932/faire-de-la-ville-un-havre-du-vivre-ensemble

Cambron-Goulet, D. (2020, 11 avril). Le Québec va changer mais la banlieue ne sera pas prise d'assaut après la crise. Journal de Québec. Repéré à www.journaldemontreal.com/2020/04/11/la-banlieue-ne-sera-pas-prise-dassaut-apres-la-crise

Côté, M., Mercier, G. et Roy, F. (2014). L’urbanisation de la campagne : motifs et options du régime québécois de protection du territoire agricole. Cabiers de géographie du Québec, 58(165), 391-409. https://doi.org/10.7202/1033011ar

Cox, W. (2006). Transport et logement à Montréal : comment le développement de la banlieue rend la métropole plus compétitive. Cabiers de l'Institut économique de Montréal, juillet 2006. Repéré à www.iedm.org/fr/541-transport-et-logement-a-montreal-commentle-developpement-de-la-banlieue-rend-la-metropole-plus-competitive

Deglise, F. (2020, 30 avril). Pourquoi la COVID-19 frappe-t-elle plus le Québec? Le Devoir. Repéré à www.ledevoir.com/ societe/sante/577941/5-raisons-qui-pourraient-expliquer-pourquoi-la-covid-19-frappe-plus-le-quebec

Desgré, M. (2012, 26 juillet). Le bungalow, patrimoine des banlieues du Québec. CitaZine. Repéré à www.citazine.fr/article/bungalowpatrimoine-banlieues-quebec

Diotte, S. (2020, 4 mars). Vivre à la verticale. L'Actualité. Repéré à https:/ / lactualite.com/societe/vivre-a-la-verticale

Fortin, A., Després, C. et Vachon, G. (dir.). (2002). La banlieue revisitée. Québec : Nota Bene.

Gonzalez, G. (2010, 29 mars). Urban sprawl, oil, and U.S. foreign policy. Paper presented at the 2010 Western Political Science Association Annual Meeting, San Francisco, CA. Repéré à https://ssrn.com/abstract=1580417

Goulet, D. (2020, 2 avril). Les grandes épidémies qui ont frappé le Québec. Québec Science. Repéré à www.quebecscience.qc.ca/sante/grandes-epidemies-quebec

Grenier, F. (2020, 1 avril). COVID-19 :la pandémie va-t-elle remettre en cause la densité urbaine? Magarine 100 degrés. Repéré à https:// centdegres.ca/magazine/sante-et-societe/la-pandemie-remettre-en-cause-densite-urbaine

Guillemette, M. (2020, 22 avril). Il va falloir désapprendre la peur de la COVID-19. Québec Science. Repéré à www.quebecscience.qc.ca/sante/desapprendre-peur-covid

Hummel, T. (2020, 2 avril). Les banlieues françaises, grandes oubliées du confinement. Carrefour International. Repéré à www.courrierinternational.com/article/vu-dallemagne-les-banlieues-francaises-grandes-oubliees-du-confinement

Jargowsky, P. A. (2002). Sprawl, concentration of poverty and urban inequality. Dans G. D. Squires (dir.). Urban sprawl: Causes, consequences, and policy responses (p. 39-72). Washington (DC): Urban Institute Press.

Klaus, I. (2020, 6 mars). Pandemics are also an urban planning problem. City Lab. Repéré à www.citylab.com/design/2020/03/ coronavirus-urban-planning-global-cities-infectious-disease/607603/

La Presse canadienne (2020, 28 avril). COVID-19 : 1000 morts à Montréal, qui recommande le port du masque. L'Actualité. Repéré à https://lactualite.com/actualites/covid-19-1000-morts-a-montreal-qui-recommande-le-port-du-masque

Leymarie, J. (2020, 27 avril). Coronavirus : «le réel allié du virus, c'est la sur-occupation des logements, la promiscuité », affirme un expert en immobilier. Franceinfo. Repéré à www.francetvinfo.fr/sante/maladie/coronavirus/coronavirus-le-reel-allie-du-virusc-est-la-sur-occupation-des-logements-la-promiscuite-affirme-un-expert-en-immobilier_3912389.html

Litzier, J.-B. (2020, 8 avril). Le confinement va-t-il doper la demande de logement en maison? Le Figaro. Repéré à https://immobilier.lefigaro.fr/article/le-confinement-va-t-il-doper-la-demande-de-logement-en-maison_3a726868-78e111ea-9e2c-96d0306afde6 
Menton, J. (2020, 1 mai). Get me out of here! Americans flee crowded cities amid COVID-19, consider permanent moves. US A Today. Repéré à www.usatoday.com/story/money/2020/05/01/coronavirus-americans-flee-cities-suburbs/3045025001/

Morisset, L. K. et Noppen, L. (2004). Le bungalow québécois, monument vernaculaire : de l'espace urbain à l’identité domestique. Cabiers de géographie du Québec, 48(134), 127-154. https://doi.org/10.7202/011678ar

Saint-Hilaire, M. (2020, 8 mai). Le télétravail pour les régions. Le Quotidien. Repéré à www.lequotidien.com/opinions/editoriaux/ le-teletravail-pour-les-regions-12f83a03f5631f2c147fa5ff5597165b

Saldivia, G. (2018, 20 septembre). Stuck in traffic? You're not alone: New data show Americans commute times are longer. NPR News. Repéré à www.npr.org/2018/09/20/650061560/stuck-in-traffic-youre-not-alone-new-data-show-american-commutetimes-are-longer

Savard, C. (2020, 15 avril). Le mois le plus long. Infolettre de Vivre en ville. Repéré à https://vivreenville.org/nos-positions/ chroniques/2020/le-mois-le-plus-long.aspx

Simard, M. (2014). Étalement urbain, empreinte écologique et ville durable : y a-t-il une solution de rechange à la densification? Cabiers de géographie du Québec, 58(165), 331-352. https://doi.org/10.7202/1033008ar

World Wildlife Fund (WWF). (2014). Living planet report 2014: Species and spaces, people and places. Gland, Suisse: World Wildlife Fund. Repéré à www.worldwildlife.org/pages/living-planet-report-2014 